\title{
Test method for empirically determining inertial properties of manual wheelchairs
}

\author{
Matthew R. Eicholtz, MS; ${ }^{1-2 *}$ Jayme J. Caspall, MS; ${ }^{1-2}$ Phuc V. Dao, MS; ${ }^{1-2}$ Stephen Sprigle, PhD, PT; ${ }^{2-3}$ \\ Al Ferri, $\mathbf{P h D}^{\mathbf{1}}$ \\ ${ }^{1}$ Department of Mechanical Engineering; ${ }^{2}$ Rehabilitation Engineering and Applied Research Laboratory, College of \\ Architecture; and ${ }^{3}$ Department of Applied Physiology, Georgia Institute of Technology, Atlanta, GA
}

\begin{abstract}
The iMachine is a spring-loaded turntable used to measure inertial properties of irregularly shaped rigid bodies, specifically manual wheelchairs. We used a Newton-Euler approach to calculate wheelchair mass and center of mass (CM) location from static force measurements using load cells. We determined the moment of inertia about the vertical axis from the natural frequency of the system in simple harmonic motion. The device was calibrated to eliminate the effects of platform components on measurement error. For objects with known inertial properties, the average relative error of the mass and the CM coordinates ( $x$ and $y$ ) were $0.76 \%, 0.89 \%$, and $1.99 \%$, respectively. The resolution of the moment of inertia calculation depends on the ratio of test piece inertia to system inertia, such that the higher the ratio, the more accurate the measurements. We conducted a Gage Repeatability and Reproducibility (Gage R\&R) test using three manual wheelchairs measured three times by three operators; the results showed that over $90 \%$ of the variance in inertia was caused by differences in the wheelchairs being measured. Gage R\&R analysis indicated that measurement system operation was acceptable using criteria from the Automobile Industry Action Group for both inertia and mass measurements.
\end{abstract}

Key words: center of mass, free vibration, iMachine, mass, mathematical models, mechanical design, moment of inertia, rigid-body dynamics, simple harmonic oscillator, wheelchair testing.

\section{INTRODUCTION}

The need for precise measurement of wheelchair inertia has arisen as part of a core effort to measure the efficiency of manual wheelchairs during the execution of certain brief maneuvers. In the proposed system, efficiency is determined by accurate measurements (within $10 \%$ ) of work input and kinetic energy output for an arbitrary manual wheelchair propelled by a robotic occupant called the Anatomical Model Propulsion System (AMPS). The AMPS is a machine with anthropomorphic mass distribution that drives the wheelchair using the push rims with a push-recovery profile similar to that of a human occupant. The estimation of kinetic energy depends on the inertial properties of the AMPS-occupied wheelchair, specifically the mass and moment of inertia. For the purposes of the efficiency calculations, the

\footnotetext{
Abbreviations: AMPS = Anatomical Model Propulsion System, CM = center of mass, DOF = degree of freedom, FFT = fast Fourier transformation, Gage $R \& R=$ Gage Repeatability and Reproducibility, GUI = graphical user interface, SD = standard deviation.

*Address all correspondence to Matthew R. Eicholtz, MS; Carnegie Mellon University-Mechanical Engineering, 5000 Forbes Ave, Scaife Hall, B-2, Pittsburgh, PA 15213; 813-731-9963; fax: 412-268-3348.

Email: mattheweicholtz@gmail.com

http://dx.doi.org/10.1682/JRRD.2011.03.0045
} 
wheelchair is constrained to the horizontal plane, and rotation about the vertical (yaw) axis is of primary interest. Therefore, the main objective of the project described here is to accurately determine the mass and moment of inertia about the yaw axis of a manual wheelchair. The initial experiment is only concerned with unoccupied manual wheelchairs because the AMPS had not yet been completed.

For simple systems with well-defined shapes and densities, inertial properties can be determined analytically using closed-form formulas. For more complex systems, it may be necessary to derive these properties empirically. Many techniques have been developed to achieve this goal, leading to several patented devices [13]. While three-dimensional modeling software is capable of estimating inertial parameters [4], modeling inaccuracies and ill-defined material properties lead to significant errors. More recently, Almeida et al. outlined a handful of modern approaches to inertia parameter identification, including "modal methods," which derive the entire inertia tensor of an object by attempting to excite it at its rigid body modes [5]. Despite these new computationally complex attempts to increase the precision with which rigid body mass properties can be measured, conventional methods using simple free vibration principles are well established and offer a sufficient amount of accuracy for most experimental applications [6-10]. The trifilar pendulum is arguably the most popular device, where pendulum cables (or files) generate a restoring torque to induce simple harmonic motion [7-9]. DuBois et al. suggest this method to be the most accurate, with reported errors of $<1$ percent [7]. However, the major challenge with the multifilar pendulum approach is centering the center of mass (CM) of the object about the rotation axis, without which errors propagate in the measurements [8]. While Hou et al. have found that strategically adding known masses to the platform can balance the forces more easily than moving a cumbersome test piece [9], perhaps an even more efficient method exists for centering the mass.

For the study presented here, the objects being measured are manual wheelchairs, for which little research exists on empirically-derived estimates of inertia [11]. Power wheelchairs have been studied, however, using a combination of force plate data with a motion capture system [12] and the traditional torsional pendulum method [13]. In the latter case, the relative error of the inertia measurement for objects with known mass properties was 9 to 10 percent.
With this in mind, we designed a device dubbed the iMachine to accurately measure the yaw inertia of manual wheelchairs using free vibration principles. However, its utility is not restricted to that purpose. It can theoretically measure the inertia of any rigid body about any axis, provided that a suitable fixture exists to mount the rigid body in the proper orientation on the machine. The ability to efficiently reposition a wheelchair on the iMachine is a design goal that would improve upon previous research efforts. Other inertial properties that can be derived from the iMachine include the mass of the wheelchair and the location of its CM. While the primary objective is to provide accurate inertia estimates for the measurement of efficiency of manual wheelchairs, a secondary outcome of this work is knowledge translation of experimental inertia identification techniques, which may improve upon or generate new studies on wheelchair performance, because inertia is an important factor in wheelchair propulsion, especially during turning maneuvers.

\section{METHODS}

\section{Measurement Approach}

Figure 1 illustrates a basic model of a spring-loaded turntable that acts as a simple harmonic oscillator. It is assumed that the system is underdamped and that the effects of a small change in damping are negligible so that the primary controllable design parameter is the springs. The three inertial parameters we are interested in measuring using the iMachine device include the mass, the location of the CM, and the moment of inertia about the yaw axis passing through the CM. The first two of these parameters can be computed under static conditions, while the moment of inertia is determined from the oscillation period.

As discussed later in the "Design Criteria" section, one of the novel requirements of the iMachine is that it must incorporate an adjustable "platform" that rigidly attaches the wheelchair to the rotating turntable. However, the presence of a platform adds to the mass and inertia of the chair. This article describes a measurement procedure that calibrates the system and corrects all measured parameters for the presence of the platform. The mass of the wheelchair $\left(m_{\mathrm{w}}\right)$ can be computed as

$$
m_{\mathrm{w}}=m_{\mathrm{pw}}-m_{\mathrm{p}},
$$


where $m_{\mathrm{pw}}=$ the mass of the platform with the wheelchair mounted on it and $m_{\mathrm{p}}=$ the mass of the platform alone. While the mass computation does not require a specific number of transducers, three transducers (labeled A, B, and C in Figure 1) are required to compute the planar location of the wheelchair's CM. Relative to the body-fixed coordinate axes shown in Figure 1, we determined the CM coordinates $(x, y)$ by summing the moments caused by the transducer forces about the $x$ - and $y$-axes, yielding Equations 2 and 3:

$$
\begin{gathered}
X=\frac{\left(F_{\mathrm{C}} d_{\mathrm{C}}-F_{\mathrm{B}} d_{\mathrm{B}}\right) \cos 30^{\circ}}{F_{\mathrm{A}}+F_{\mathrm{B}}+F_{\mathrm{C}}} \text { and } \\
Y=\frac{F_{\mathrm{A}} d_{\mathrm{A}}-\left(F_{\mathrm{B}} d_{\mathrm{B}}+F_{\mathrm{C}} d_{\mathrm{C}}\right) \sin 30^{\circ}}{F_{\mathrm{A}}+F_{\mathrm{B}}+F_{\mathrm{C}}},
\end{gathered}
$$

where $F=$ force measured by each transducer and $d=$ distance to transducer. The design intent was to place the transducers at equal distances from the turntable axle in order to simplify these expressions, but in practice, we measured the actual distances for each transducer to

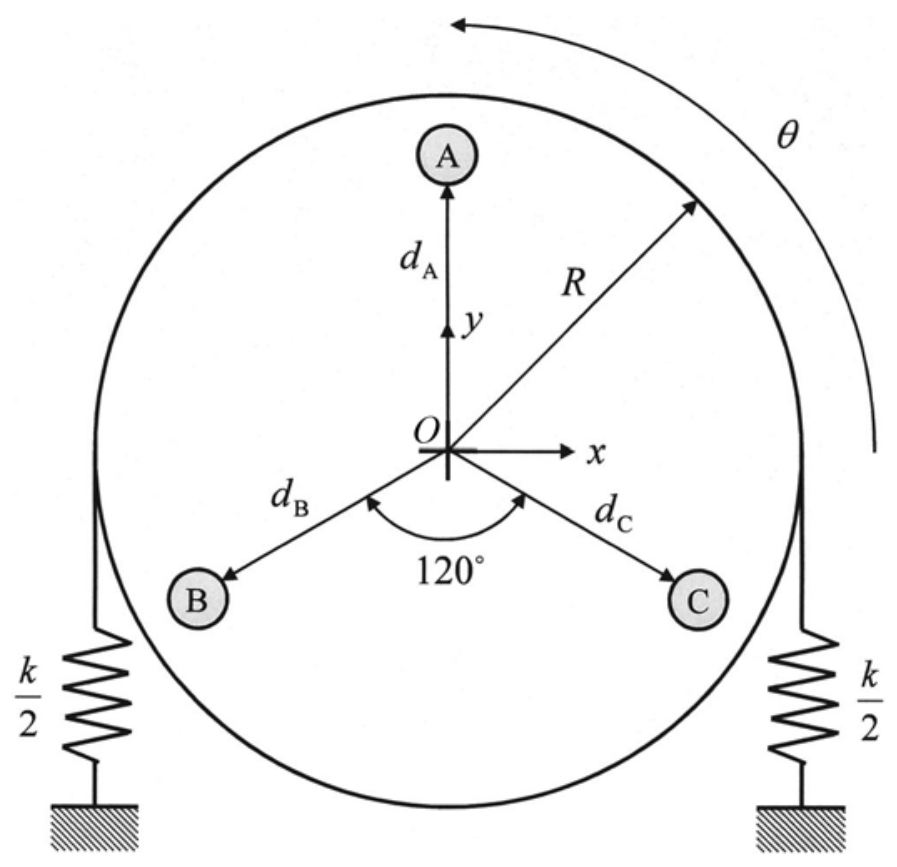

Figure 1.

Model of iMachine design. $\theta=$ angle of oscillation, $A=$ transducer $\mathrm{A}, \mathrm{B}=$ transducer $\mathrm{B}, \mathrm{C}=$ transducer $\mathrm{C}, d=$ distance, $k=$ spring stiffness, $O=$ center point, $R=$ radius, $x=x$-axis, $y=$ $y$-axis. reduce computational error. If, after mounting the wheelchair, the CM coordinates estimated by Equations 2 and $\mathbf{3}$ are nonzero, the wheelchair should be repositioned to drive these coordinates to approximately zero. Note, however, that this results in the CM for the wheelchairplatform combination being centered at point $O$; the $\mathrm{CM}$ of the wheelchair will, in general, be off the rotation axis, necessitating the corrections described later to find the centroidal mass-moment of inertia of the wheelchair alone.

For the dynamic portion of the test, we gave the total system an initial angular displacement with an initial angular velocity equal to zero. In the system under consideration, the generalized coordinate is the angular position of the turntable, such that summing the moments about the turntable axle yields the following equation of motion:

$$
I \ddot{\theta}+c \dot{\theta}+k R^{2} \theta=0,
$$

where $I=$ the moment of inertia to be measured, $\theta=$ angular position, $\dot{\theta}=$ angular velocity (first time derivative of position), $\ddot{\theta}=$ angular acceleration (second time derivative of position), $k=$ overall spring stiffness, and $R=$ the moment-arm of the spring force, which is equal to the radius of the rotating disk by design. The parameter $c$ is an unknown but small, viscous damping coefficient that accounts for a variety of loss mechanisms, including bearing friction. Dividing through by the inertia, Equation 4 takes the general form

$$
\ddot{\theta}+2 \zeta \omega_{\mathrm{n}} \dot{\theta}+\omega_{\mathrm{n}}^{2} \theta=0
$$

where $\omega_{\mathrm{n}}=\sqrt{\left(k \mathrm{R}^{2}\right) / \mathrm{I}}$ is the natural frequency and $\zeta=$ the viscous damping ratio; then the moment of inertia is calculated using the relationship

$$
I=\frac{k R^{2}}{\omega_{\mathrm{n}}^{2}} .
$$

The natural frequency is related to the natural period of oscillation, $T_{\mathrm{n}}=2 \pi / \omega_{\mathrm{n}}$, which can be measured during the dynamic test. Several time domain methods exist for estimating the period of oscillation, including counting successive zeros, maxima, and minima. We can also approximate the natural frequency by analyzing angular position data in the frequency domain. The moment of inertia computed using Equation 6 reflects that of the entire system, i.e., the wheelchair and the platform. It is necessary to remove the 
wheelchair and run the dynamic test again to extract the wheelchair inertia, maintaining the configuration of the platform. We calculated the appropriate inertia by taking the difference between the two tests:

$$
\left(I_{\mathrm{zz}}^{\mathrm{O}}\right)_{\mathrm{w}}=\left(I_{\mathrm{zz}}^{\mathrm{O}}\right)_{\mathrm{pw}}-\left(I_{\mathrm{zz}}^{\mathrm{O}}\right)_{\mathrm{p}},
$$

where $z=$ axis of rotation. However, as mentioned previously, the analysis is not yet complete because in general, point $O$ in this calculation is not on the vertical axis passing through the wheelchair CM. Using the parallel-axis theorem, we calculated the desired moment of inertia by

$$
\left(I_{\mathrm{zz}}\right)_{\mathrm{w}}=\left(I_{\mathrm{zz}}^{\mathrm{O}}\right)_{\mathrm{w}}-m_{\mathrm{w}} d_{\mathrm{w}}^{2}
$$

where the distance $d_{\mathrm{w}}$ is given by the relationship $d_{\mathrm{w}}^{2}=x_{\mathrm{w}}^{2}+y_{\mathrm{w}}^{2}$, and each component of the CM offset is computed using the following relationships:

$$
\begin{aligned}
& x_{\mathrm{w}}=-\frac{m_{\mathrm{p}}}{m_{\mathrm{w}}} x_{\mathrm{p}} \quad \text { and } \\
& y_{\mathrm{w}}=-\frac{m_{\mathrm{p}}}{m_{\mathrm{w}}} y_{\mathrm{p}},
\end{aligned}
$$

where $\left(x_{\mathrm{p}}, y_{\mathrm{p}}\right)$ are the calculated values of the platform CM obtained during the second dynamic test after removing the wheelchair. The equations used to compute these coordinates resemble Equations 2 and 3, replacing $(x, y)$ with $\left(x_{\mathrm{p}}, y_{\mathrm{p}}\right)$.

\section{Design Criteria}

The fundamental design criterion for the inertia measurement device is the accurate measurement of the desired inertia parameters using the approach described previously. The relative error of the inertia measurement should be 10 percent to achieve results that are comparable with similar research efforts conducted previously. The test method should be both repeatable and robust. Along these lines, in order to maintain rigid body dynamics, we judiciously set the maximum frequency of oscillation to $1 \mathrm{~Hz}$, which reduces the probability of component "slop" adding one or more degrees of freedom (DOFs) to the system. Furthermore, we set the maximum load capacity of the device at $136 \mathrm{~kg}$ (300 lb) to account for an occupied manual wheelchair, specifically for a manual wheelchair occupied by the AMPS. These two quantitative specifications guide machine design decisions regarding spring stiffness, platform geometry, and load support mechanisms (e.g., shaft and bearings). Another important design requirement is the development of a simple and effective method for repositioning the wheelchair on the turntable, which is an improvement over previous studies by increasing test productivity and reducing measurement error [8]. Other specifications that we considered during the design phase include reducing the profile and footprint of the machine and ensuring ease of assembly and use.

\section{Evaluation Techniques}

\section{Calibration}

In order to increase the overall accuracy of the inertia measurement device, we calibrated the linear springs in situ so that any uncertainty in the normal operation of the machine would be taken into account in the determination of the spring rate. To accomplish this test, we placed two diametrically opposed mass blocks with similar, known inertia properties on the test platform. When we executed the dynamic test, the measured system inertia corresponded to the sum of the test platform inertia and twice the block inertia. We computed the block inertia using the parallel-axis theorem, such that

$$
\left(I_{\text {zz }}^{\mathrm{O}}\right)_{\text {block }}=\frac{m_{\text {block }}}{12}\left(l^{2}+w^{2}\right)+m_{\text {block }}\left(s+\frac{w}{2}\right)^{2},
$$

where $m_{\text {block }}=$ the block mass, $l$ and $w=$ the length and width of the block, respectively, and $s=$ the perpendicular distance from the axis of rotation to the edge of the block. Using Equation 6, the relationship between the effective spring rate of the system ( $k_{\text {eff }}$, equal to twice the stiffness of each spring) and the inertia components is given by

$$
\frac{k_{\text {eff }} R^{2}}{\omega_{\mathrm{n}}^{2}}=\left(I_{\mathrm{zz}}^{\mathrm{o}}\right)_{\mathrm{p}}+2\left[\frac{m_{\text {block }}}{12}\left(l^{2}+w^{2}\right)+m_{\text {block }}\left(s+\frac{w}{2}\right)^{2}\right],
$$

with $\omega_{\mathrm{n}}$ determined from an experiment; $k_{\text {eff }}$ can be obtained from Equation 12 only if the platform inertia is known. Unfortunately, we must determine the test platform inertia empirically because its irregular shape does 
not lend itself to theoretical computation using simple closed-form equations. By executing the test at two different distances, $s_{1}$ and $s_{2}$, the effective spring rate in Equation 12 can be canceled by taking the ratio

$$
\frac{\omega_{1}^{2}}{\omega_{2}^{2}}=\frac{\left(I_{\mathrm{zz}}^{\mathrm{O}}\right)_{\mathrm{p}}+\frac{m_{\text {block }}}{6}\left(l^{2}+w^{2}\right)+2 m_{\text {block }}\left(s_{2}+\frac{w}{2}\right)^{2}}{\left(I_{\mathrm{zz}}^{\mathrm{O}}\right)_{\mathrm{p}}+\frac{m_{\text {block }}}{6}\left(l^{2}+w^{2}\right)+2 m_{\text {block }}\left(s_{1}+\frac{w}{2}\right)^{2}} .
$$

Equation 13 can be rearranged to solve for the inertia of the test platform, which is then substituted back into Equation 12 to calculate the effective spring rate. We performed the test with the blocks at distances $s_{1}=50.8 \mathrm{~mm}$ ( $2 \mathrm{in}$.) and $s_{2}=76.2 \mathrm{~mm}$ ( 3 in.). We tested each configuration 20 times for reliability and used both time-domain and frequency-domain techniques to estimate the natural frequency of oscillation.

\section{Validation Testing}

To validate the measurements of the test method described previously, we conducted controlled tests using mass blocks with known mass and theoretically calculated moment of inertia. We compared mass measurements recorded by the iMachine with the corresponding mass given by a commercially available scale with a resolution of $0.02 \mathrm{lb}$. To validate the iMachine's ability to determine the CM coordinates, we successively placed a stack of blocks on the test platform at equally spaced intervals along the $x$ - and $y$-axes and compared empirical measurements with theoretical predictions. For moment of inertia validation, we arranged the mass blocks in a symmetrical pattern on the test platform to facilitate the theoretical calculation of the system moment of inertia. We tested multiple block configurations in order to characterize the effect of inertia variability on measurement error and determine which frequency estimation technique achieves the best results.

\section{Gage Repeatability and Reproducibility Testing}

To assess the reliability of the test method for measuring the inertial properties of manual wheelchairs, we performed a Gage Repeatability and Reproducibility (Gage $\mathrm{R} \& \mathrm{R})$ test. This analysis of variance measurement technique is used to compare the variability of the wheelchair's inertial properties with the variability of the test method itself, including Gage error (repeatability) and operator error (reproducibility). For this study, we measured three different wheelchairs three times each with three different operators. The wheelchair models included a Quickie GT (Sunrise Medical; Longmont, Colorado), Quickie GTX (Sunrise Medical), and E\&J Advantage (Graham-Field Health Products, Inc; Atlanta, Georgia). We selected these specifically because of differences in frame style, size, and material construction. Table 1 shows descriptions of wheelchair parameters.

Each test replication of a wheelchair was considered independent, meaning the chair was completely removed from the platform and the system brought back to an initial arbitrary configuration after each test. The test sequence was randomized for each operator to reduce the effect of bias caused by learning or improving operator

Table 1.

Characteristics of manual wheelchairs used in Gage Repeatability and Reproducibility testing.

\begin{tabular}{|c|c|c|c|}
\hline Characteristic & & Wheelchair & \\
\hline Model & Quickie GT* & Quickie GTX $^{*}$ & E\&J Advantage $^{\dagger}$ \\
\hline Frame Material & Aluminum & Aluminum & Steel \\
\hline Frame Type & Rigid & Folding & Folding \\
\hline Wheels and Tires & $\begin{array}{l}\text { Spoke wheels with } \\
\text { pneumatic tires }\end{array}$ & $\begin{array}{l}\text { Spoke wheels with } \\
\text { pneumatic tires }\end{array}$ & $\begin{array}{l}\text { Mag wheels with } \\
\text { solid tires }\end{array}$ \\
\hline Caster Diameter (cm) & 10 & 10 & 20 \\
\hline Front Track (cm) & 45 & 50 & 48 \\
\hline Wheelbase (cm) & 42 & 44 & 42 \\
\hline
\end{tabular}


technique. Test operators were given appropriate literature to read, including a standard operating procedures document for the machine. In addition, under the guidance of a researcher who was very familiar with the test method, each operator was afforded a practice session consisting of one to two tests to familiarize themselves with the process and further reduce learning bias.

\section{RESULTS}

\section{Machine Design}

The selected design is a spring-loaded turntable that is free to oscillate in the horizontal plane about its vertical axle. Figure 2 illustrates the final prototype of the iMachine. Two linear extension springs (McMaster-Carr; Sante Fe Springs, California), each with a stiffness of $588 \mathrm{~N} / \mathrm{m}$ (value provided by manufacturer), generate the necessary torque to allow free vibration of the system. The turntable is comprised of a $59.7 \mathrm{~cm}$-diameter composite disk mounted to a stepped axle that rotates with respect to a custom aluminum collar and extruded aluminum frame assembly (80/20 Inc; Columbia City, Indiana) that is fixed to the ground. The collar holds two ball bearings that support the radial and axial loads on the axle, reduce the frictional effects on rotation, and restrict the tilt of the platform. An optical encoder (E3 series, U.S. Digital; Vancouver, Washington) fixed to the bottom of the shaft records the angular position of the turntable in real time. The encoder resolution is proportional to the maximum allowable angular speed by the equation, $\omega_{\max }=f_{\min } \Delta \theta$, where $f_{\min }=$ the minimum sampling rate. We used a resolution of $0.036^{\circ}$ and a sampling rate of $2,500 \mathrm{~Hz}$, which yield a maximum allowable angular speed of $\pi / 2 \mathrm{rad} / \mathrm{s}$. Three miniature compression load cells (LCGB series, Omega Engineering, Inc; Stamford, Connecticut) with a load capacity of $113.4 \mathrm{~kg}$ ( $250 \mathrm{lb}$ ) each are mounted to the top of the turntable at positions $\mathrm{A}, \mathrm{B}$, and $\mathrm{C}$ shown in
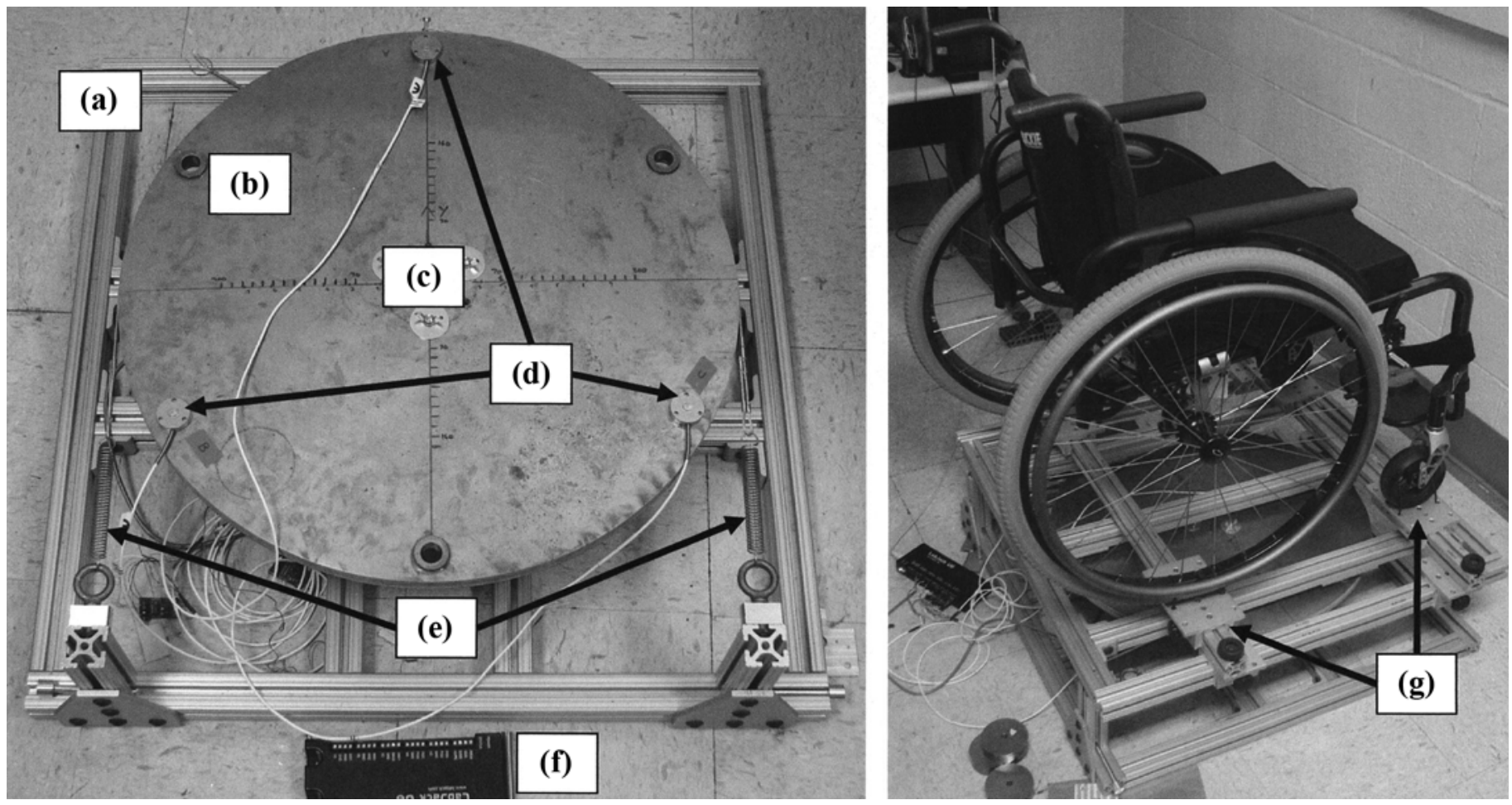

\section{Figure 2.}

iMachine shown without positioning platform (left) and with Quickie GT manual wheelchair (Sunrise Medical; Longmont, Colorado) mounted to device with positioning platform included (right). System components include (a) bottom structural frame, (b) composite rotating disk, (c) shaft/bearing/encoder assembly mounted to bottom of disk and connected to structural frame (not visible), (d) three load cells, (e) two linear extension springs, (f) LabJack U6 data acquisition device (LabJack Corporation; Lakewood, Colorado), and (g) $x-y$ positioning platform with linear bearings for each set of wheels. 
Figure 1. The interface between the load cells and the test piece is an $x-y$ positioning platform made from extruded aluminum beams and linear bearings (80/20 Inc). Data acquisition was accomplished using a LabJack U6 device (LabJack Corporation; Lakewood, Colorado) and a custom graphical user interface (GUI) developed in LabVIEW (National Instruments Corporation; Austin, Texas). Data postprocessing was performed using MATLAB algorithms (The MathWorks, Inc; Natick, Massachusetts).

\section{Calibration}

Figures 3 and 4 illustrate example plots of the angular position in the time and frequency domains, respectively. For the former, the natural frequency is the inverse of twice the half-period of oscillation, which we estimated by counting zero crossings, successive maxima, or successive minima. For the latter, we computed the fast Fourier transformation (FFT) via MATLAB, which makes use of the FFTW ("Fastest Fourier Transform in the West") library that is based on the Cooley-Tukey FFT algorithm [14-15], and used standard MATLAB functions (i.e., find, max) to identify the natural (dominant) frequency. All of the natural frequency estimation methods used in the calibration procedure were very precise, resulting in standard deviations (SDs) of $<0.00869 \mathrm{rad} / \mathrm{s}$ ( $0.16 \%$ of the mean). Nonetheless, the FFT achieved the best results, with computed average natural frequencies of $5.520 \mathrm{rad} / \mathrm{s}$ and $5.478 \mathrm{rad} / \mathrm{s}$ for the distance $s$ of $50.8 \mathrm{~mm}$ and $76.2 \mathrm{~mm}$, respectively $(n=20)$. As a result, the aver-

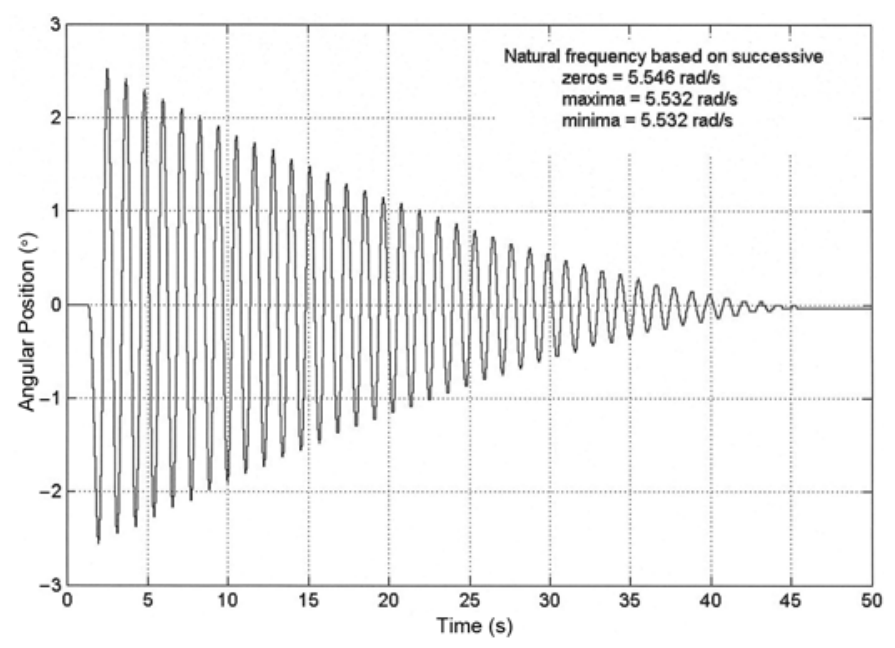

Figure 3.

Example plot of angular position in time domain from calibration test $($ distance $=50.8 \mathrm{~mm})$.

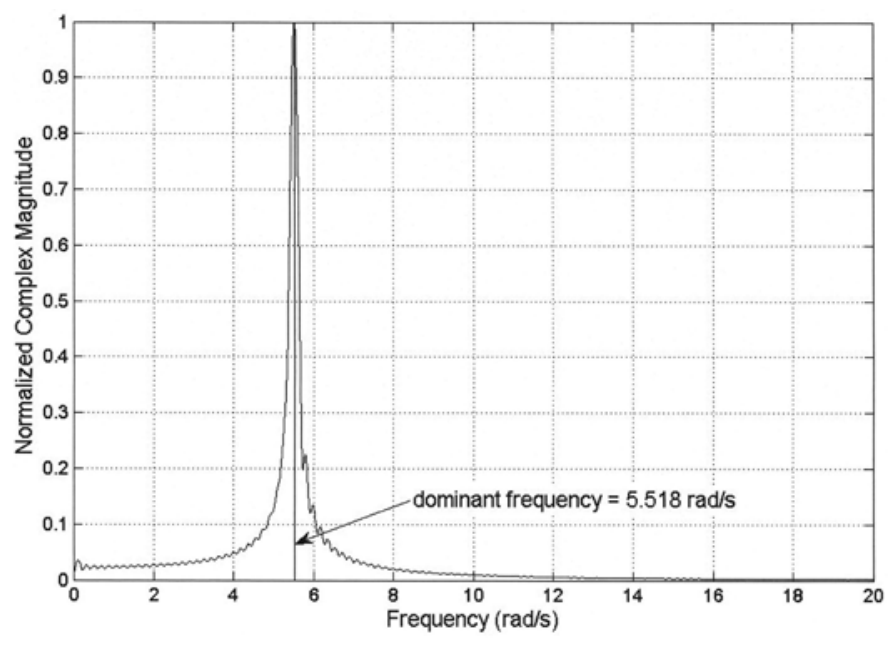

Figure 4.

Example plot of angular position in frequency domain from calibration test $($ distance $=50.8 \mathrm{~mm})$.

age empirical moment of inertia for the test platform equaled $3.452 \mathrm{~kg}-\mathrm{m}^{2}$. By back-substituting these values into Equation 12 along with the moment arm of the spring force, which equaled the radius of the disk $(29.85 \mathrm{~cm})$, we computed an effective spring rate for each test run. For the frequency-domain method, the average effective spring rates for the two test configurations were (mean $\pm \mathrm{SD}) 1,231.087 \pm 2.628 \mathrm{~N} / \mathrm{m}(s=50.8 \mathrm{~mm})$ and $1,231.088 \pm 2.696 \mathrm{~N} / \mathrm{m}(\mathrm{s}=76.2 \mathrm{~mm})$. By comparison, the manufacturer-provided spring rate is $1,176.850 \mathrm{~N} / \mathrm{m}$, indicating a relative difference of 4.61 percent.

\section{Validation Testing}

During mass validation, we varied the system mass from 19.00 to $44.60 \mathrm{~kg}$, which covers the range of masses expected from the unoccupied manual wheelchairs used in the study. The average relative percent error of the mass measurements was 0.762 percent, with an absolute error range from 0.00 to $0.40 \mathrm{~kg}$. For the CM validation, the average relative error across all test configurations was 0.891 and 1.990 percent for the $x$ - and $y$-coordinates, respectively.

Regarding moment of inertia validation, only the FFT-based results are presented here because the aforementioned tests indicated this method was the most accurate. We evaluated two different applied masses with the iMachine configured with and without the $x-y$ positioning platform. The first test centered a single mass block on the turntable $\left(I_{\mathrm{th}}=0.0298 \mathrm{~kg}-\mathrm{m}^{2}\right.$, where $I_{\mathrm{th}}=$ theoretical 
moment of inertia calculated from the geometry and mass of the object) without using the $x-y$ positioning platform. The natural frequency measurements exhibited good repeatability $(N=30)$, with an SD of $0.00757 \mathrm{rad} / \mathrm{s}(0.1 \%$ of the mean). Using the measurement approach described previously, the empirical inertia of the block about its $\mathrm{CM}$ was $0.0368 \mathrm{~kg}-\mathrm{m}^{2}$, yielding a relative error $>23$ percent when compared against theoretical predictions. The second test configuration was obtained by reattaching the $x-y$ positioning platform to the turntable to reflect nominal operation of the device. We arranged four similar mass blocks in a square configuration about the rotation axis, with the perpendicular distance between the axis of rotation and the edge of each block equal to $0.152 \mathrm{~m}$ (6 in.). We computed the theoretical inertia of the 4-brick arrangement to be $I_{\mathrm{th}}=0.918 \mathrm{~kg}-\mathrm{m}^{2}$. The average peak frequency $(n=5)$ according to the FFT was $5.036 \mathrm{rad} / \mathrm{s}$ for the case with the test objects and $5.622 \mathrm{rad} / \mathrm{s}$ when the objects were removed. We computed the average inertia of the test objects about the CM as $0.854 \mathrm{~kg}-\mathrm{m}^{2}$, which corresponds to a relative error of 6.96 percent.

The results of the first two inertia tests motivated a third test configuration (see "Discussion" section), which involved the same four-block square configuration as before, but positioned on the turntable without the $x-y$ positioning platform. For this test, the average peak frequency $(n=5)$ was $9.043 \mathrm{rad} / \mathrm{s}$ for the case with the test objects and $16.037 \mathrm{rad} / \mathrm{s}$ when the objects were removed. As a result, we determined the average inertia of the test configuration about its $\mathrm{CM}$ to be $0.916 \mathrm{~kg}-\mathrm{m}^{2}$, yielding the lowest relative error in the validation study $(<0.25 \%)$.

\section{Gage Repeatability and Reproducibility Testing}

Table 2 tabulates the result of the Gage R\&R test and

Figures $\mathbf{5}$ and $\mathbf{6}$ show plots of the mass and moment of inertia measurements. The Gage R\&R analysis reports the amount of variation caused by the measurement sys- tem and the difference between parts, which in this case is the different wheelchair models. Furthermore, measurement system variation is divided into two components. Repeatability reflects the variability from repeated measurements of the same part by the same operator and reproducibility reflects the variability when the same part is measured by different operators.

The results indicate that the measurement system accounted for 7.95 percent of the study variance when measuring the moment of inertia and 3.90 percent when measuring wheelchair mass. The part-to-part differences contributed over 99 percent of the variance of both metrics.

\section{DISCUSSION}

The iMachine test results indicated that we the met primary design criterion of accurately measuring inertial properties of manual wheelchairs. Regarding the measurement approach, we explored several techniques for estimating the period of oscillation, including counting successive zeros, maxima, and minima and analyzing the angular position data in the frequency domain. However, controlled tests using objects of known inertia revealed that the greatest accuracy is achieved by the latter method. Therein, we performed an FFT on the data and assumed the dominant frequency to be approximately equal to the natural frequency of the system. The effect of damping was neglected by studying the narrowness of the maximum peak, or quality factor, which showed damping to be $<2$ percent in general.

Concerning the mechanical design, all components met the required design specifications, including load capacity and size. Specifically, the composite disk and shaft assembly that comprise the turntable was designed to accommodate wheelchair widths up to $61 \mathrm{~cm}$ (24 in.) with a maximum load of $136 \mathrm{~kg}$ (300 lb) applied at the edge of

Table 2.

Gage Repeatability and Reproducibility (Gage R\&R) moment of inertia and mass of three different chairs measured by three different operators three times each.

\begin{tabular}{|c|c|c|c|c|}
\hline \multirow{2}{*}{ Source of Variation } & \multicolumn{2}{|c|}{ Moment of Inertia } & \multicolumn{2}{|c|}{ Mass } \\
\hline & Contribution (\%) & Study Variance (\%) & Contribution (\%) & Study Variance (\%) \\
\hline Total Gage R\&R & 0.63 & 7.95 & 0.15 & 3.90 \\
\hline Repeatability & 0.63 & 7.95 & 0.12 & 3.51 \\
\hline Reproducibility & 0.00 & $\mathbf{0 . 0 0}$ & 0.03 & 1.71 \\
\hline Part-to-Part & 99.37 & 99.68 & 99.85 & 99.92 \\
\hline
\end{tabular}

Note: Bold numbers emphasize moment of inertia results, which were more important to this study than mass results. 


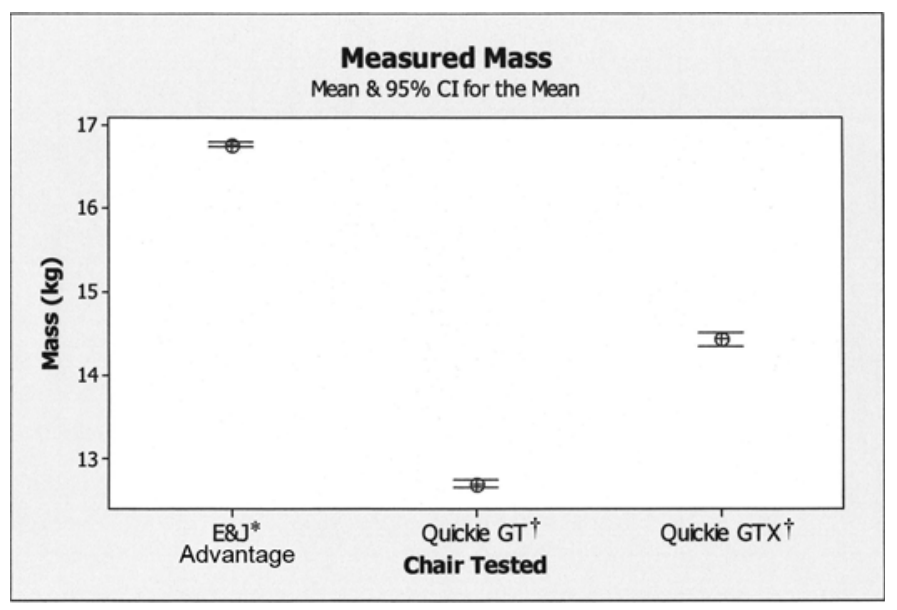

Figure 5.

Gage Repeatability and Reproducibility results for measured manual wheelchair mass. $\mathrm{Cl}=$ confidence interval.

*Graham-Field Health Products, Inc; Atlanta, Georgia.

†Sunrise Medical; Longmont, Colorado.

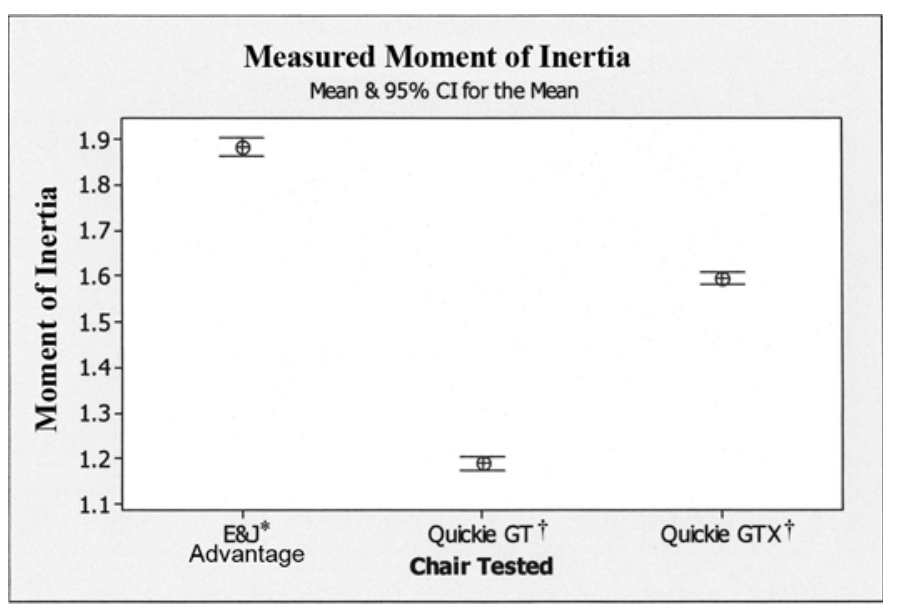

Figure 6.

Gage Repeatability and Reproducibility results for measured manual wheelchair moment of inertia. $\mathrm{Cl}=$ confidence interval. *Graham-Field Health Products, Inc; Atlanta, Georgia.

†'Sunrise Medical; Longmont, Colorado.

the disk, taking into account the possibility that the wheelchair may be occupied. The springs were designed to prohibit geometric interference with other system components and meet the frequency constraint. Although this study used a stiffness of $588 \mathrm{~N} / \mathrm{m}$ (value provided by manufacturer) for each spring, the springs are easily interchangeable to accommodate variable stiffness based on desired frequency specifications and anticipated inertia of the object being measured. Another major component for meeting the design criteria is the $x-y$ positioning platform. It allows the test object to be easily repositioned so that the $\mathrm{CM}$ can be made collinear with the axis of rotation, thus eliminating a parallel-axis term in the computational analysis and making the machine easier to use in practice. The load capacity and resolution of the force transducers proved to be sufficient for the project goals during validation testing. In these tests, mass blocks that were less than one-third of the maximum anticipated load of an occupied wheelchair (136 kg) exhibited >99 percent accuracy on average, and relative percent error should theoretically decrease with increasing load. For the optical encoder, we hypothesized that the encoder resolution and selected sampling rate would be sufficient for ensuring that the angular position is measured properly given small rotations. Controlled tests verified this hypothesis and showed that only $2^{\circ}$ to $5^{\circ}$ of rotation was needed to generate a sufficient number of oscillations in the angular position signal.

Another benefit of the test method used here is the software approach for conducting and analyzing experiments. The custom LabVIEW GUI (Figure 7) allows the experimenter to view the test piece mass, CM coordinates, and angular position using intuitive gauges and plots that update in real time. Additional user controls include the ability to start, stop, and record a test and modify geometric, transducer, and data streaming parameters. We completed post hoc data processing using a simple set of MATLAB algorithms that can be easily changed to accommodate desired statistical measures. A written set of standard operating procedures complement the user interface and data processing functions, which makes the iMachine easy to use, even for a researcher unfamiliar with the technical aspects of the device.

During validation testing, we found the accuracy of the iMachine to correlate to the inertia ratio, which is defined as the ratio of the inertia of the test piece to the inertia of the whole system. Figure 8 shows the plot of the correlation. As the inertia ratio increases, the relative error in the measurement decreases. Since the main application of the iMachine is to measure wheelchair inertia, the inertia ratio is expected to be much higher than the mass blocks used in validation testing. Consequently, the relative error in the measurement of wheelchair moment of inertia is expected to be in the range of 5 to 10 percent. Based on these observations, it may be beneficial in future studies to reduce the inertia of the turntable and $x-y$ positioning platform. This can be accomplished by using 


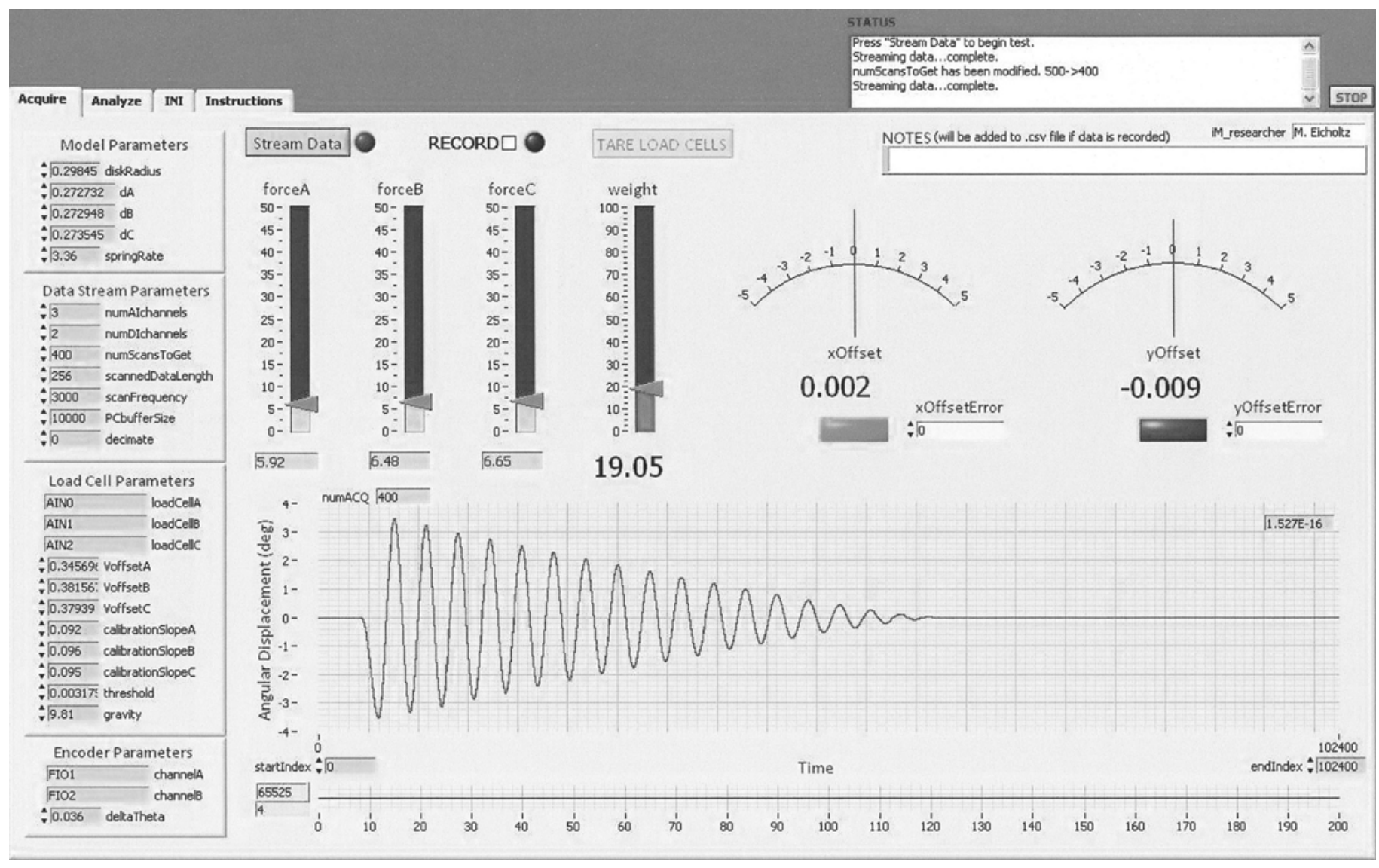

Figure 7.

LabVIEW (National Instruments Corporation; Austin, Texas) front panel of iMachine graphical user interface.

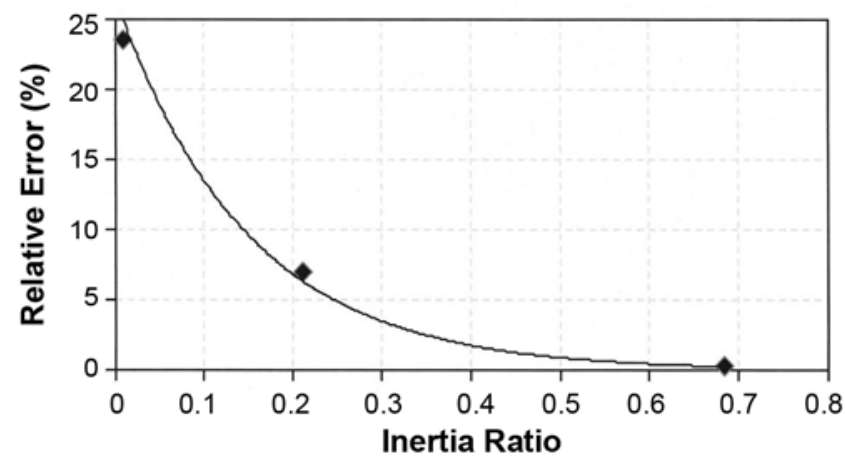

Figure 8.

Plot of relative error versus inertia ratio (ratio of test piece inertia to total inertia of test piece and turntable).

lighter materials or changing the shape to reduce the mass, while maintaining strength requirements. Additionally, further testing on objects corresponding to varying inertia ratios may elucidate the relationship presented in the aforementioned figure and provide better error estimates for the inertia measurement device.

The results of the crossed Gage R\&R analysis indicate that the measurement system (iMachine) operation was acceptable using criteria from the Automobile Industry Action Group [16] for both inertia and mass measurements. For inertia measurement, the total Gage R\&R, i.e., the variation of the measurement system, was about 8 percent and for mass was 4 percent. By extension, this Gage R\&R result indicates that $>99$ percent of the variance was caused by the parts (different wheelchair models) and speaks to the robustness of the test method presented here.

This test method is applicable, within certain limitations, to almost any object for which inertial properties need to be determined. The main limitations of the iMachine, in particular, are load capacity, anticipated inertia, and mounting ability. While the system is currently intended only for manual wheelchairs, it is capable of 
measuring inertial properties for any wheelchair (manual or powered) that weighs less than $136 \mathrm{~kg}$ (300 lb). For loads greater than this limit, mechanical design changes need to be made to the main platform. The measurement accuracy is limited by the ratio of the test piece inertia to the system inertia. In its current setup, the inertia of the $x$-y positioning platform and turntable is $3.452 \mathrm{~kg}-\mathrm{m}^{2}$, such that the inertia of the test piece needs to be $>1.15 \mathrm{~kg}-\mathrm{m}^{2}$ to ensure 95 percent accuracy, according to Figure 8. If the anticipated inertia of the test piece is less than this threshold, relative error will be high unless modifications are made to the iMachine to reduce its inertia. The measurement approach is based on rigid-body dynamics, and measurement errors propagate if the test piece behaves as a flexible body. However, if the test piece can be mounted to the $x-y$ positioning platform in a secure manner for varying orientations, it is theoretically possible to determine the moment of inertia about any axis.

\section{CONCLUSIONS}

The goal of this study was to design and investigate a test method for empirically measuring inertia properties of manual wheelchairs. To that end, the theoretical dynamics of a 1-DOF rigid-body system in simple harmonic motion motivated the design of the iMachine, a spring-loaded rotating platform capable of withstanding loads up to $136 \mathrm{~kg}$ (300 lb) and angular speeds up to $\pi / 2 \mathrm{rad} / \mathrm{s}$. The mass measurement exhibited $>99$ percent accuracy on average, while the measured CM coordinates had an average accuracy of 98 percent. The accuracy of the moment of inertia measurement depended on the proportion of the system inertia represented by the test piece. As the inertia of the test piece increased relative to the platform, the measurement accuracy also increased. The wheelchairs that we tested accounted for approximately 25 percent of the system inertia, and tests on objects with known mass properties show this case should have errors of $<5$ percent. This is an improvement over previously published results from similar studies. While future studies may seek to optimize the machine design in order to minimize platform inertia and thereby reduce measurement error, the results presented in this article indicate a device of this nature is a reliable and robust option for empirically determining inertial properties of manual wheelchairs and other irregularly-shaped rigid bodies.

\section{ACKNOWLEDGMENTS}

\section{Author Contributions:}

Study concept and design: M. R. Eicholtz, J. J. Caspall, P. V. Dao, S. Sprigle.

Acquisition of data: M. R. Eicholtz.

Analysis and interpretation of data: M. R. Eicholtz, S. Sprigle.

Drafting of manuscript: M. R. Eicholtz.

Critical revision of manuscript for important intellectual content:

M. R. Eicholtz, J. J. Caspall, P. V. Dao, S. Sprigle, A. Ferri.

Statistical analysis: S. Sprigle.

Obtained funding: S. Sprigle.

Administrative, technical, or material support: M. R. Eicholtz,

J. J. Caspall, P. V. Dao, S. Sprigle, A. Ferri.

Study supervision: J. J. Caspall, S. Sprigle, A. Ferri.

Financial Disclosures: The authors have declared that no competing interests exist.

Funding/Support: This material is the result of work supported in part by the Mobility Rehabilitation Engineering Research Center, National Institute on Disability and Rehabilitation Research, U.S. Department of Education (grant H133E080003).

Additional Contributions: The authors would like to acknowledge Stanley Wang for his contribution to building the device and collecting data. Mr. Eicholtz is currently with the Department of Mechanical Engineering, Carnegie Mellon University, Pittsburgh, Pennsylvania.

Disclaimer: The opinions contained in this article are those of the grantee and do not necessarily reflect those of the U.S. Department of Education.

\section{REFERENCES}

1. Boynton Richard S, inventor. Moment of inertia measuring instrument. United States patent US 3,693,413. 1972 Sep 26.

2. Carpenter David A, inventor; MRC Corporation, assignee. Mass properties measurement system. United States patent US 4,161,876. 1979 Jul 24.

3. Mastinu Gianpiero, Doniselli Carlo, inventors; Search USPTO Assignment Database, assignee. Device for measuring the inertia tensor of a rigid body. United States patent US 7,278,295. 2007 Oct 9.

4. Pegram JP, Anemaat WA. Preliminary estimation of airplane moments of inertia using CAD solid modeling. SAE Paper 2000-01-1700. 2000.

5. Almeida RA, Urgueira AP, Maia NM. Further developments on the estimation of rigid body properties from experimental data. Mech Sys Signal Processing. 2010;24(5):1391-1408. http://dx.doi.org/10.1016/j.ymssp.2009.12.003

6. Ogata K. System dynamics. 4th ed. Upper Saddle River (NJ): Pearson/Prentice Hall; 2004.

7. Du Bois JL, Lieven NA, Adhikari S. Error analysis in trifilar inertia measurements. Exper Mech. 2009;49(4):533-40. http://dx.doi.org/10.1007/s11340-008-9142-4 
8. Ringegni PL, Patanella AJ. An experimental technique for determining mass inertial properties of irregular shape bodies and mechanical assemblies. Measurement. 2001;29(1): 63-75. http://dx.doi.org/10.1016/S0263-2241(00)00028-2

9. Hou ZC, Lu Y, Lao Y, Liu D. A new trifilar pendulum approach to identify all inertia parameters of a rigid body or assembly. Mechanism Machine Theory. 2009;44(6):1270-80. http://dx.doi.org/10.1016/j.mechmachtheory.2008.07.004

10. Griffiths IW, Watkins J, Sharpe D. Measuring the moment of inertia of the human body by a rotating platform method. Am J Physics. 2005;73(1):85-93. http://dx.doi.org/10.1119/1.1648688

11. Kauzlarich JJ, Thacker JG. A theory of wheelchair wheelie performance. J Rehabil Res Dev. 1987;24(2):67-80. [PMID: 3585785]

12. Ding D, Cooper RA, Guo S, Corfman TA. Analysis of driving backward in an electric-powered wheelchair. IEEE Trans Control Sys Technol. 2004;12(6):934-43. http://dx.doi.org/10.1109/TCST.2004.833638

13. Wang H, Grindle GG, Connor S, Cooper RA. An experimental method for measuring the moment of inertia of an electric power wheelchair. Conf Proc IEEE Eng Med Biol Soc. 2007;2007:4798-4801. [PMID: 18003079]

14. FFTW [Internet]. Cambridge (MA): FFTW. Available from: http://www.fftw.org
15. Frigo M, Johnson SG. FFTW: An adaptive software architecture for the FFT. Proc Int Conf Acoustics Speech Signal Proc. 1998;3:1381-84.

16. DaimlerChrysler, Ford Motor Company, General Motors Corporation. Measurement systems analysis: Reference manual. Detroit (MI): DaimlerChrysler; Ford Motor; General Motors; 2002.

Submitted for publication March 23, 2011. Accepted in revised form July 26, 2011.

This article and any supplementary material should be cited as follows:

Eicholtz MR, Caspall JJ, Dao PV, Sprigle S, Ferri A. Test method for empirically determining inertial properties of manual wheelchairs. J Rehabil Res Dev. 2012;49(1):5162.

http://dx.doi.org/10.1682/JRRD.2011.03.0045

ResearcherID: Matthew R. Eicholtz, MS: A-2822-2011

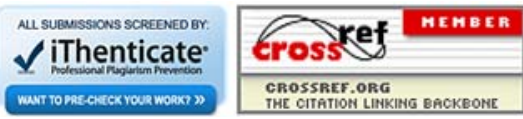

